\title{
Sistemas de Informação Geográfica (SIG) como ferramenta de apoio para a gestão de projetos ambientais
}

\section{Gabriela Diedrichs Barbosa*, Maria Helena da Fonseca}

Universidade Tecnológica Federal do Paraná (UTFPR). Av. Monteiro Lobado, s/n, km 04. Jardim Carvalho. Ponta Grossa-PR. Brasil. (CEP 84016-210). *E-mail: gabrieladiedrichsbarbosa@gmail.com.

Resumo. Este trabalho busca explorar a utilização de Sistemas de Informação Geográfica (SIG) como ferramenta empregada para apoiar a gestão de projetos ambientais. O SIG é uma ferramenta de geoprocessamento amplamente utilizada para análises espaciais de dados georreferenciados. Seu uso inclui estudos de mapeamento, diagnósticos, avaliações de impactos e alterações na superfície terrestre, monitoramento, controle e ordenamento territorial. Para a realização deste trabalho, a metodologia foi baseada em pesquisa bibliográfica, onde foi possível perceber o emprego cada vez mais frequente de SIG em projetos ambientais em todo o mundo. $\mathrm{O}$ presente artigo apresenta trabalhos que possuem como base de sua metodologia algum software de SIG para gerenciar projetos ambientais diversificados. Os SIG podem ser eficientemente utilizados para auxiliar a gestão de projetos ambientais nos mais diversos setores e organizações, auxiliando a visualização de dados, permitindo análises espacializadas e contribuindo na gestão, no planejamento e nas tomadas de decisão dos responsáveis pela gestão de projetos.

Palavras-chave: Gestão de projetos; Gestão ambiental; Sistemas de Informação Geográfica.

Abstract. Geographic Information Systems (GIS) as a support tool for the environmental projects management. This paper seeks to explore the use of geographic information systems (GIS) as a tool used to support the management of environmental projects. GIS is a widely used geoprocessing tool for spatial analysis of georeferenced data. The use of GIS includes mapping studies, diagnostics, impact assessments and land surface changes, monitoring, control and land use planning. For the accomplishment of this paper, the methodology was based on bibliographical research. It was possible to see the increasing use of GIS in environmental projects around the world. The present paper presents works that have as base of their methodology some GIS software to manage diversified environmental projects. GIS can be efficiently used to assist the management of environmental projects in various sectors and organizations, helping to visualize data, allowing spatialized analysis and contributing to the management, planning and decision making of project managers.

Keywords: Project management; Environmental management; Geographic Information Systems.

Recebido:

$14 / 11 / 2016$

Aceito:

03/09/2016

Publicado:

30/06/2017

Acesso Aberto

Artigo completo

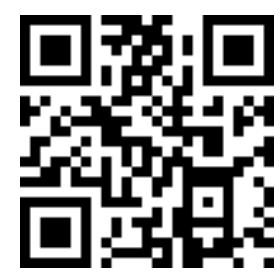

ORCID

(1) 0000-0003-2027-460X

Gabriela Diedrichs

Barbosa

D 0000-0001-8369-0980

Maria Helena da

Fonseca 


\section{Introdução}

Projetos devem ser gerenciados de forma que apresentem seus resultados dentro do prazo e orçamento anteriormente previstos (PMI, 2008), pois são eles que viabilizam o alcance dos objetivos das empresas e organizações (Reis, 2015). Tendo em vista a competitividade de mercado cada vez mais acentuada, uma gestão eficiente de um projeto é um diferencial para qualquer setor, uma vez que auxilia tomadas de decisão e resolução de problemas (Chagas et al., 2015).

Um dos grandes desafios encontrado em todo o mundo é a busca por soluções para os problemas ambientais, tema amplamente discutido e que, por consequência, faz crescer a quantidade de projetos que tem como base os aspectos da sustentabilidade ambiental, cujos objetivos são encontrar recursos para mitigar os impactos negativos causados pelo homem ao meio ambiente.

Tais interferências humanas sobre o meio ambiente demandam por tecnologias que auxiliem a sua gestão, manejo e monitoramento ambiental. Isso resulta na constante evolução tecnológica dos sistemas de informação geográfica, uma ferramenta cada vez mais utilizada para dar suporte na gestão de projetos (Madeiros e Câmara, 2001).

Assim, o presente trabalho teve como objetivo discutir o uso de sistemas de informação geográfica (SIG), uma ferramenta do geoprocessamento amplamente utilizada para análises espaciais de dados georreferenciados, no auxílio à gestão de projetos ambientais em diferentes setores e organizações, de maneira que haja contribuição e apoio nas tomadas de decisões dos gestores responsáveis e promova a sustentabilidade. Para isso, foi utilizada como metodologia a pesquisa bibliográfica, realizada nas principais bases de dados nacionais e internacionais.

\section{Gestão de projetos}

Projeto é um conjunto de atividades com escopo, recursos, início e fim pré- definidos, realizadas em grupo, destinadas a produzir um produto, serviço ou resultados únicos, de forma a atingir um objetivo específico (PMI, 2008).

Gerenciamento de projetos consiste na aplicação de conhecimentos, habilidades e técnicas para a execução de projetos de forma efetiva e eficaz. O gerenciamento eficiente de projetos é uma competência estratégica para as organizações, permitindo a união de resultados dos projetos com os objetivos do negócio, melhorando, assim, a competição em seus mercados (PMI, 2008).

O ciclo de vida de um projeto é composto por cinco fases: iniciação, planejamento, execução, monitoramento e controle e, por último, encerramento (Reis, 2015), descritas a seguir.

A primeira fase é a iniciação, onde a equipe e o gestor devem buscar conhecer as restrições de qualidade, de tempo, de custo e quaisquer outras influências que afetam a realização e sucesso do projeto. Um bom exemplo de documento que se usa nessa fase é o termo de abertura (Reis, 2015).

A segunda fase é o planejamento, onde há um nível de detalhamento muito maior, de forma a estruturar um plano consistente que leve o projeto ao sucesso. Os documentos que contemplam essa fase são a Estrutura Analítica de Projeto (EAP), o cronograma do projeto e os planos de gerenciamento de riscos, de comunicações e de qualidade (Reis, 2015).

A terceira fase é a execução, a qual consiste em realizar as atividades planejadas da melhor forma possível. É comum que nessa fase ocorram mudanças, como solicitações de alteração no escopo (Reis, 2015).

Paralelamente à execução ocorre o monitoramento e controle, que é a quarta fase. Nesta fase é possível garantir que o que está sendo feito é compatível com o planejado, aferindo o desempenho e o progresso em contraste com o plano (Reis, 2015).

Por fim, a quinta fase é de Encerramento, onde é oficializada a conclusão do projeto. Um documento que se usa nessa fase é o termo de aceite (Reis, 2015). 
Realizar um gerenciamento eficiente dos projetos torna a empresa mais competitiva, mais ágil, mais dinâmica e pronta para entregar muito mais valor a seus clientes. A falta de uma gestão eficiente de um projeto pode contribuir para que uma empresa não sobreviva à crescente competitividade do mercado (Reis, 2015).

\section{Projetos ambientais}

Existem diversos tipos de projetos ambientais, os quais visam a mitigação, manutenção ou melhoria da qualidade ambiental e, consequentemente, da qualidade de vida das pessoas (Cavalcanti, 1994). Eles são fundamentados em aspectos econômicos, sociais e ambientais, prestigiando a geração de renda, a inclusão social e, principalmente, a preservação do meio ambiente (AMCHAM, 2016).

Os projetos ambientais utilizam de recursos naturais ou resíduos para desenvolver novos mecanismos, criar produtos sustentáveis, gerar energia alternativa, entre outros (YSA, 2009). A otimização de processos para redução da demanda de água, o tratamento de águas residuais e efluentes, a redução de perdas, o reuso, a reciclagem, o controle de poluição, a gestão e manejo de áreas de proteção, a recuperação de áreas degradadas e a educação ambiental são alguns exemplos desses projetos que contribuem com o meio ambiente (AMCHAM, 2016).

No que diz respeito à legislação brasileira, o Projeto de Lei $n^{\circ} 5.974 / 2005$, ainda a espera de aprovação, prevê incentivos fiscais para projetos ambientais. De acordo com a proposta, as pessoas físicas e jurídicas poderão deduzir uma porcentagem do Imposto de Renda através de doações feitas a entidades sem fins lucrativos que promovam projetos de preservação do meio ambiente e uso sustentável de recursos naturais.

Em contrapartida, enquanto a referida lei não é aprovada, o Ministério do Meio Ambiente (MMA) apoia programas e projetos que buscam conhecer, proteger, recuperar e usar de forma sustentável os recursos naturais, além de promover 0 desenvolvimento de produtos e processos voltados para a conservação e o uso sustentável dos biomas brasileiros (MMA, 2016).

\section{Sistemas de Informação Geográfica}

O geoprocessamento é um instrumento tecnológico que contribui com a percepção da realidade e auxilia na definição de ações (MMA, 2016). Ele é constituído por um conjunto de tecnologias de coleta, tratamento, manipulação e apresentação de informações espaciais, voltado para um objetivo específico, possuindo como principal ferramenta os Sistemas de Informação Geográfica (SIG), ou Geographical Information System (GIS), em inglês (Câmara e Davis, 2004).

Os SIG se caracterizam pela coleta e processamento de dados espaciais obtidos a partir de levantamentos de campo, mapas, fotogrametria e sensoriamento remoto; armazenamento, recuperação, atualização e correção dos dados processados; e, manipulação e análise dos dados armazenados. Dessa forma, os SIG possuem capacidade de estabelecer relações espaciais entre elementos gráficos, sendo este o sistema mais adequado para realizar análise espacial de dados geográficos (Câmara e Davis, 2004).

Os softwares de SIG mais conhecidos e utilizados são QGis, gvSIG, ArcGIS, Spring, TerraView, IDRISI, GRASS, MapWindow e SAGA GIS, os quais diferem entre si pela disponibilidade, custo e ferramentas (Capterra, 2016; Gisgeography, 2016; Environment and Ecology, 2016).

No que diz respeito ao geoprocessamento para estudos ambientais, podem ser empregadas duas metodologias. A primeira é baseada em localizações pontuais, a qual utiliza da combinação de atributos descritivos das variáveis geográficas, onde cada localização é independente. A segunda metodologia é baseada na definição de áreas homogêneas, onde uma área corresponde a uma unidade (Madeiros e Câmara, 2001). 


\section{Usos do SIG}

A pesquisa foi realizada nas principais bases de dados internacionais e mostra que o uso de softwares de SIG como ferramenta de apoio para a gestão de projetos ambientais é cada vez mais frequente. Alguns destes estudos estão descritos a seguir.

Anguinolfi et al. (2013) desenvolveram um modelo para otimizar a reciclagem de resíduos sólidos, baseado no uso integrado de um Sistema de Apoio à Decisão (DSS) e um software de SIG. O modelo é focado na dinâmica de coleta dos materiais, onde as variáveis de estudo são a quantidade de resíduos que se encontram em cada contentor de resíduos por dia, a quantidade de material recolhida em cada área por dia e as rotas dos veículos. A aplicação do modelo foi realizada em Cogoleto, município localizado no noroeste da Itália, onde os contentores de resíduos para reciclagem foram georreferenciados no mapa através de um software de SIG (Figura 1). Como resultado, os autores verificaram que a gestão dos resíduos realizada através do modelo por ele proposto traz benefícios 2,5 vezes maiores do que a prática atual.

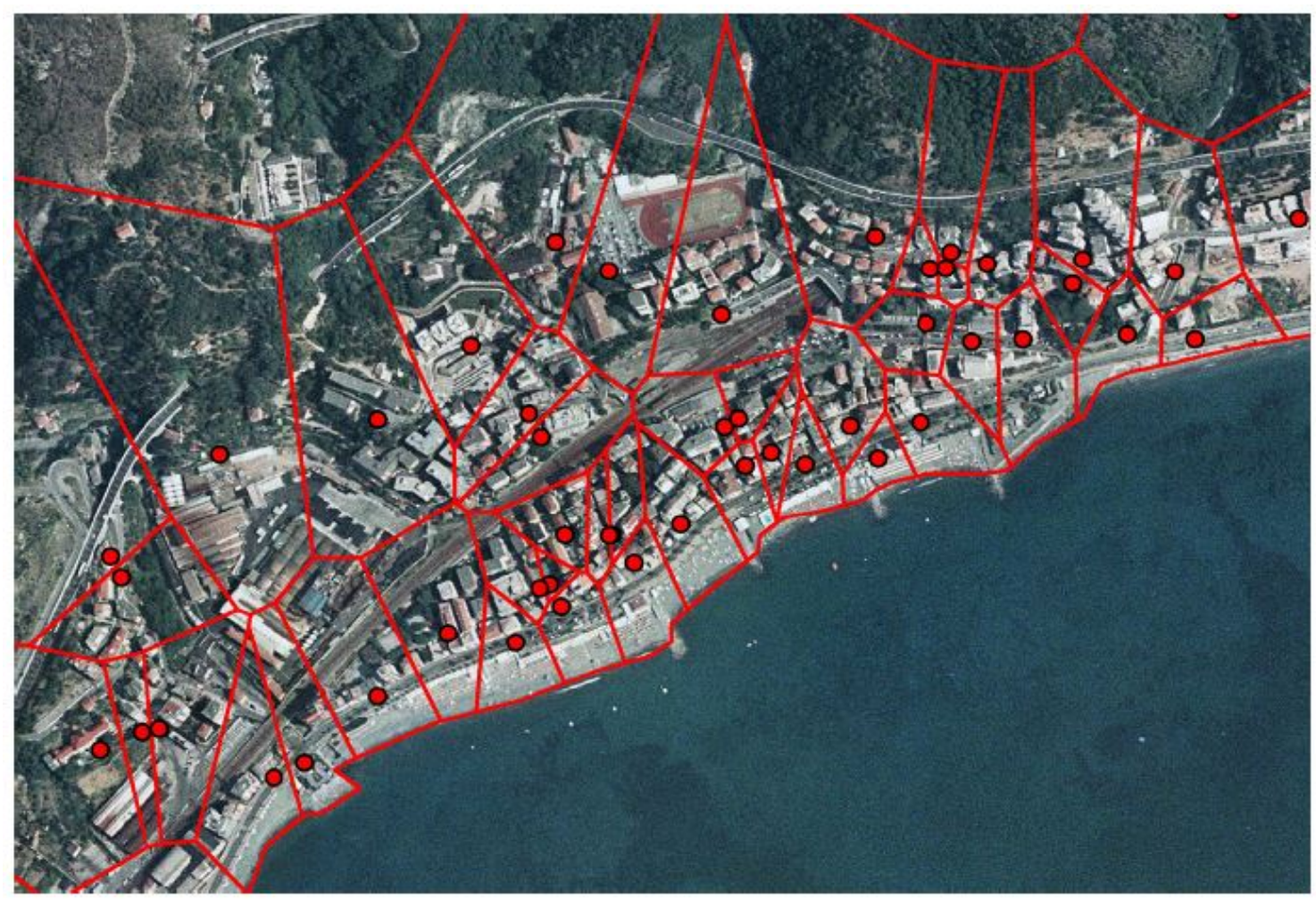

Figura 1. Georreferenciamento dos contentores de resíduos para reciclagem. Fonte: Anguinolfi et al. (2013).

Vafaeipour et al. (2014) avaliaram 25 cidades localizadas no Irã a fim de identificar as regiões adequadas para a implementação de projetos de geração de energia solar. Para isso, os autores aplicaram uma metodologia de multicritério para tomada de decisão e, por sequência, mapearam tais regiões através de um software de SIG (Figura 2), para proporcionar uma melhor visualização e identificação das prioridades de cada cidade e suas localizações geográficas. Tal abordagem auxiliou decisores do setor de energia no gerenciamento e planejamento da geração de energia em um nível superior de exigências. 


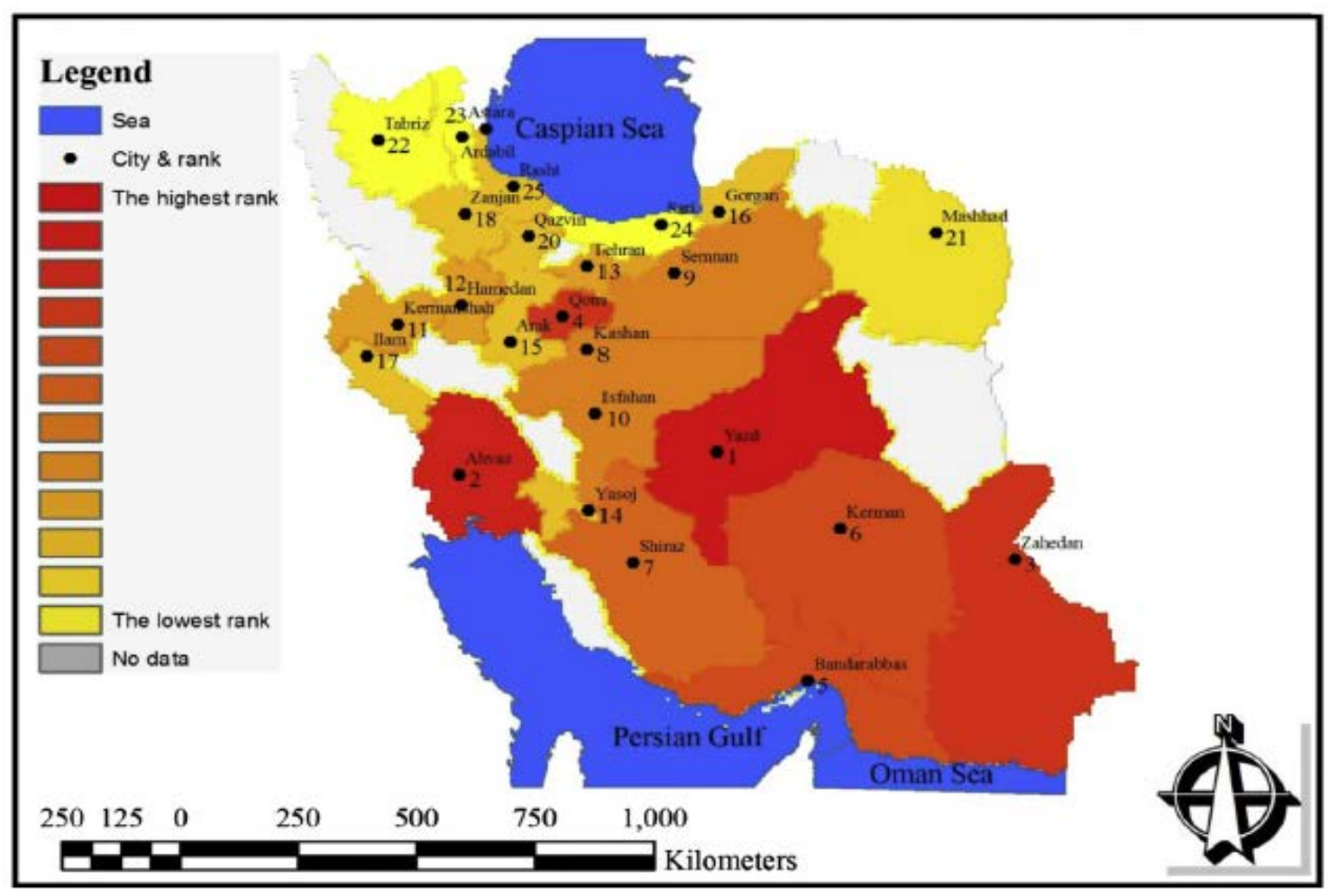

Figura 2. Mapeamento das regiões para implementação de projetos de geração de energia solar. Fonte: Vafaeipour et al. (2014).

Pactwa et al. (2015) estudaram o uso de SIG como ferramenta para apoiar a gestão de recursos minerais. O trabalho foi realizado em Swidnicki e Wroclawski, dois municípios localizados na Polônia. A modelagem das regiões com potenciais conflitos e a análise da distribuição espacial das áreas de origem e demanda dos recursos minerais foram realizados nos softwares de SIG ArcGIS, QGIS e no software distribuído pela General Public License (Figura 3). Os autores constataram que a ferramenta pode ser usada pelas autoridades responsáveis pela gestão desse material a fim de planejar os projetos de gestão dos recursos minerais disponíveis, levando em consideração os custos e benefícios de acordo com o que prevê o desenvolvimento sustentável.

Leman et al. (2016) avaliaram e classificaram áreas ambientalmente sensíveis do arquipélago Langkawi, na Malásia, para o planejamento do uso da terra da região. O estudo foi baseado na utilização de um software de SIG, ArcGIS, por meio do qual houve a geração de mapas da distribuição espacial das áreas de sensibilidade ambiental (Figura 4). Através destes, é possível fornecer informações que permitem promover o ordenamento do território e o desenvolvimento de estratégias para as áreas de sensibilidade.

Vacca et al. (2014) desenvolveram uma metodologia para mapear solos e apoiar projetos de planejamento do uso da terra em Sardenha, uma ilha da Itália que é extremamente exposta a problemas ambientais devido a sua posição geográfica e eventos climáticos. Os mapas foram criados através de dados de cobertura do solo, geologia e topografia, por meio do software de SIG ArcGIS (Figura 5). Como resultado, os autores construíram um mapa das unidades de terra e capacidade do solo da Sardenha. Tal metodologia forneceu uma maneira rápida e robusta para permitir a identificação das unidades de terra e contribuir com o planejamento do território. 


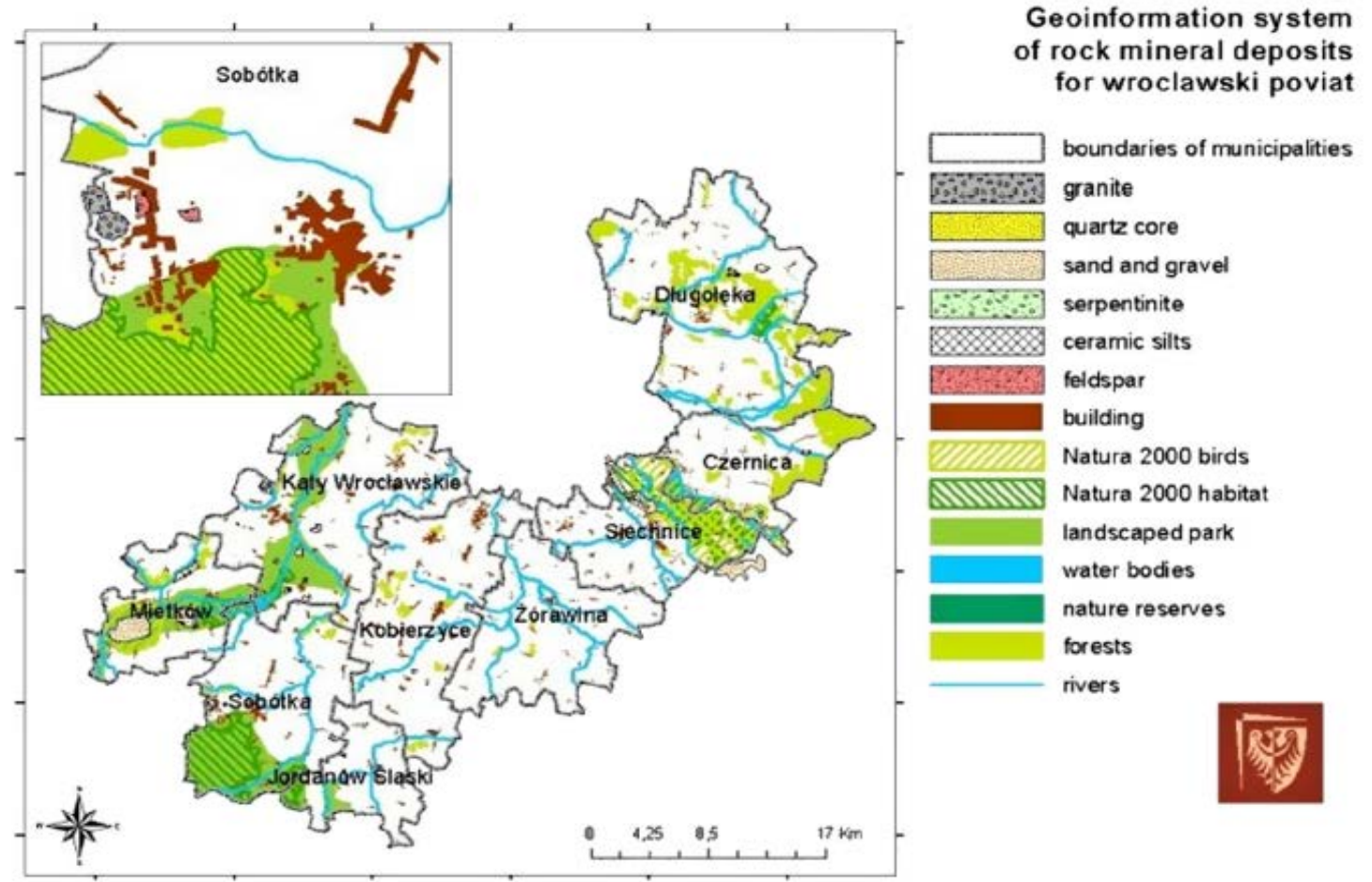

Figura 3. Mapeamento das localizações de recursos minerais em Wroclawski. Fonte: Pactwa et al. (2015).

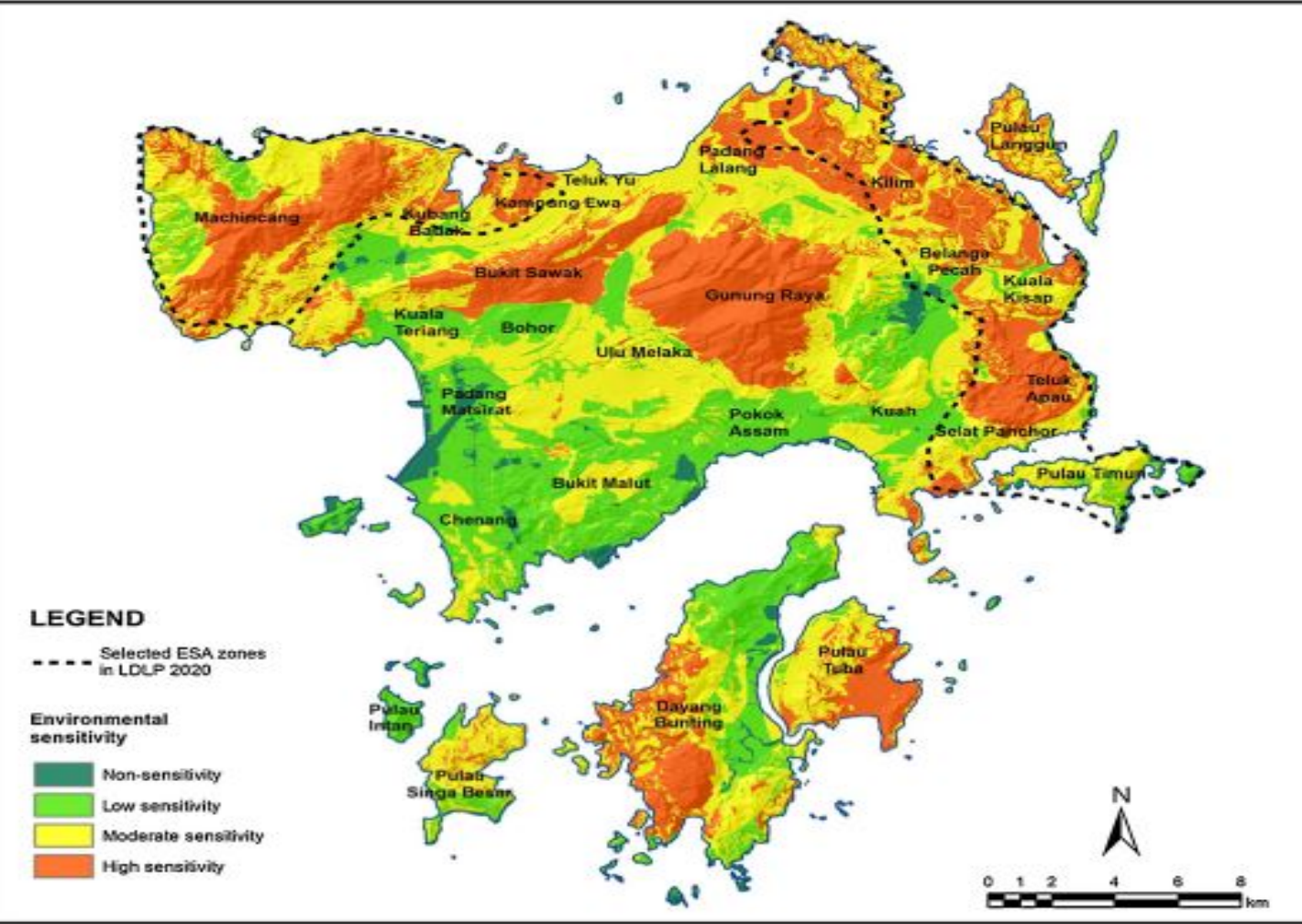

Figura 4. Áreas de sensibilidade ambiental em Langkawi. Fonte: Leman et al. (2016). 


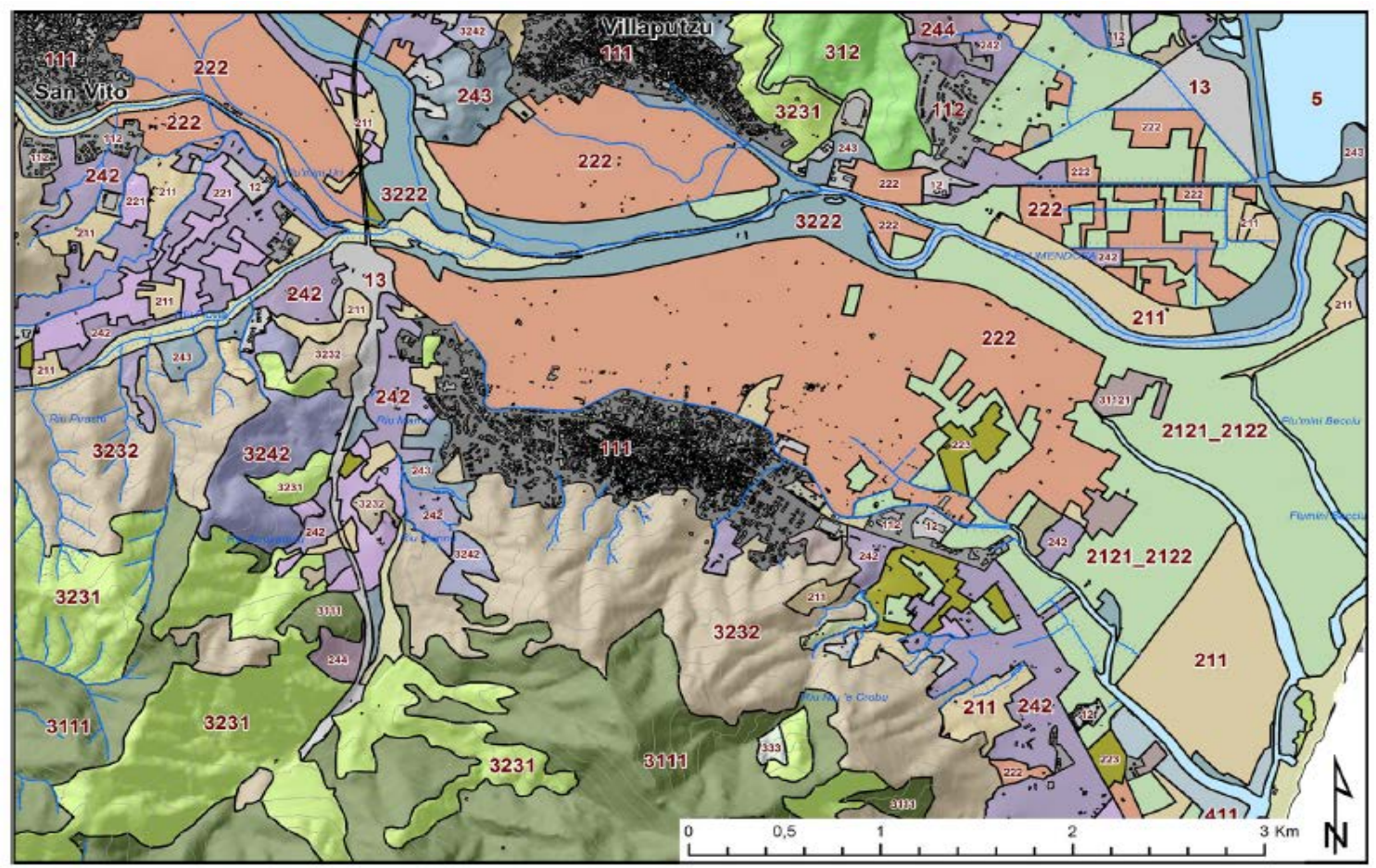

Figura 5. Mapa do uso do solo em Sardenha. Fonte: Vacca et al. (2014).

Mahboubi et al. (2015) desenvolveram, aplicaram e avaliaram um método para identificar os locais socioecologicamente importantes para 0 ambiente marinho. $\mathrm{O}$ estudo foi realizado em uma comunidade costeira no norte da Columbia Britânica, no Canadá. O software de SIG utilizado foi o xGIS, no qual foram avaliados valores socioecológicos encontrados no local, como a biodiversidade, a cultura e os valores econômicos. A partir das regiões encontradas nos mapas, foram realizadas análises estatísticas que resultou na localização espacial e na quantificação da importância dos espaços socioecológicos para o ambiente marinho (Figura 6). Os autores afirmam que o software xGIS se mostrou uma ferramenta útil para auxiliar gestores envolvidos no planejamento e gestão de projetos de recursos marinhos.

Madeiros e Câmara (2001) afirmam que as ações que envolvem a gestão de projetos ambientais incluem diversas análises, onde pode ser observado o grande impacto do uso das tecnologias de SIG. Estudos de mapeamento, os quais visam à caracterização e à compreensão da organização do espaço; diagnósticos ambientais, que estabelecem estudos específicos das regiões de interesse; avaliações de impacto ambiental, onde há levantamento e monitoramento dos resultados da intervenção humana sobre o ambiente; e trabalhos de ordenamento territorial, os quais objetivam a gestão do território de maneira sustentável; são exemplos de projetos que utilizam SIG como ferramenta para o seu gerenciamento. Tal discurso vai de encontro com a literatura, o que pode ser observado nos estudos apresentados neste tópico do artigo (Anguinolfi et al., 2013; Vafaeipour et al., 2014; Pactwa et al., 2015; Leman et al., 2016; Vacca et al., 2014; Mahboubi et al., 2015), os quais utilizam os SIG para estes fins. 


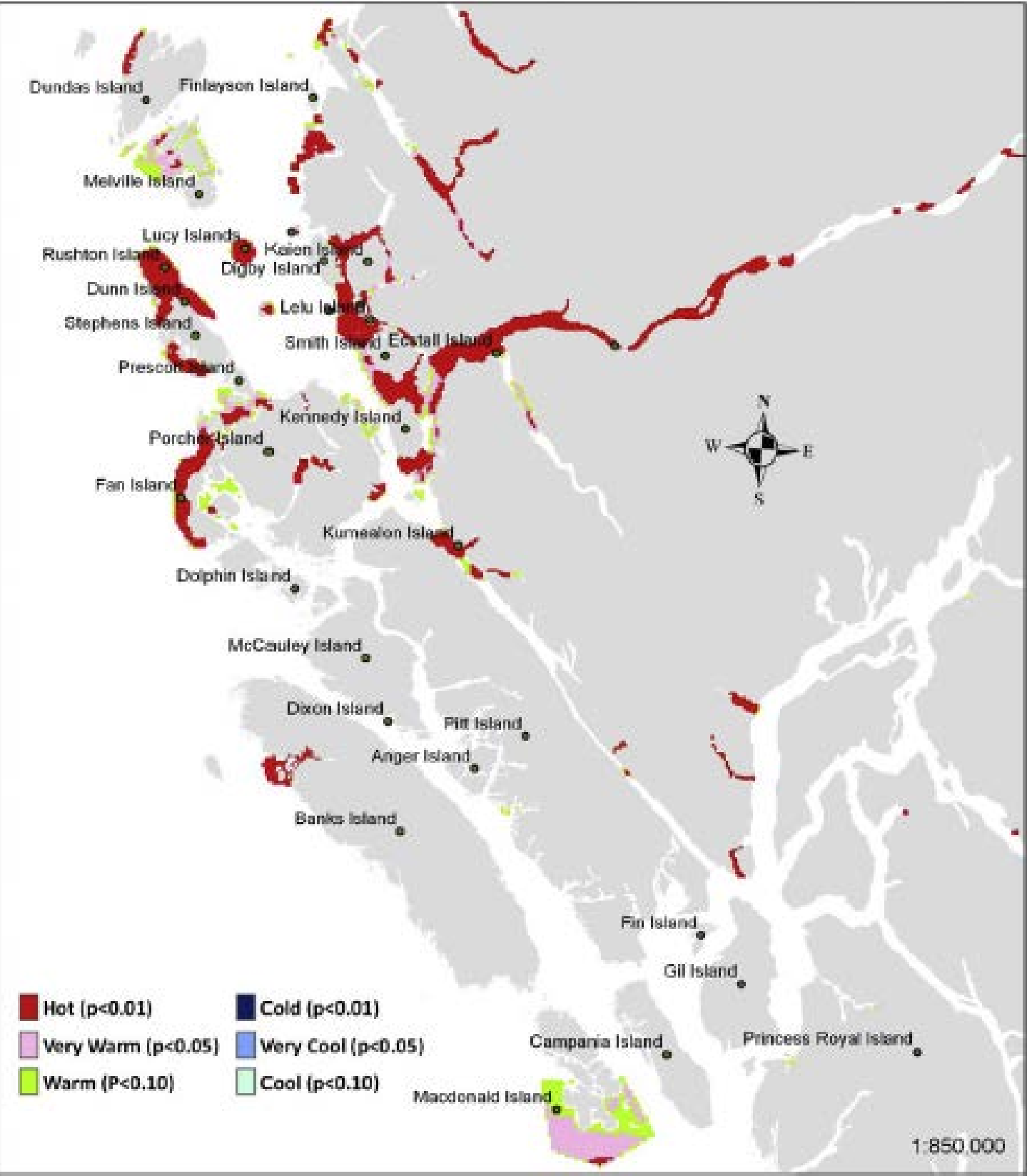

Figura 6. Mapa dos locais socioecologicamente importantes para o ambiente marinho. Fonte: Mahboubi et al. (2015).

\section{Considerações finais}

O presente trabalho discute como o uso de sistemas de informação geográfica (SIG), uma ferramenta do geoprocessamento amplamente utilizada para análises espaciais de dados georreferenciados, pode auxiliar na gestão de projetos ambientais.

Os softwares de SIG auxiliam a visualização de dados, permitindo análises espacializadas. Dessa forma é possível ter uma visão geral da área estudada, criar diferentes mapas, localizar regiões de interesse, classificar áreas, otimizar rotas, monitorar projetos, entre várias outras utilidades.

Em suma, uma metodologia baseada em ambiente SIG contribui de forma efetiva no planejamento e nas tomadas de decisão da gestão de projetos 
ambientais de diferentes setores e organizações.

\section{Referências}

AMCHAM - Câmara de Comércio Americana. Prêmio Brasil Ambiental. Rio de Janeiro. 2016. Disponível em: <http://www.amchamrio.com. br/site-evento?evento.id=224>. Acesso em: 10 ago. 2016.

Anguinolfi, D.; Paolucci, M.; Robba, M.; Taramasso, A.C. A dynamic optimization model for solid waste recycling. Waste Management, v. 33, p. 287-296, 2013.

Brasil. Projeto de Lei ${ }^{0}$ 5.974/2005. Dispõe sobre incentivos fiscais para projetos ambientais. Disponível em: $<$ http://www.camara.gov.br/proposicoesWeb/fic hadetramitacao?idProposicao=301799>. Acesso em: 12 ago. 2016.

Capterra Inc. Top GIS Software Products. Arlington, VA, USA. 2016. Disponível em: $<$ http://www.capterra.com/gis-software/>.

Acesso em: 16 ago. 2016.

Cavalcanti, C. Desenvolvimento e Natureza: estudos para uma sociedade sustentável. Recife: Instituto de Pesquisas Sociais, Fundação Joaquim Nabuco, Ministério da Educação, 1994. Disponível em: <http://168.96.200.17/ar/ libros/brasil/pesqui/cavalcanti.rtf $>$. Acesso em: 10 ago. 2016.

Chagas, R. A.; Gomes, T. C.; Lima, A. Gestão de projetos através da aplicação do método Dmaic: um estudo de caso em um processo da indústria automotiva. Anais do V Congresso Brasileiro de Engenharia de Produção, 2015, Ponta Grossa, 2015.

Environment and Ecology. List of geographic information systems software. Disponível em: $<$ http://environment-ecology.com/geographicalinformation-systems-gis/582-list-of-geographicinformation-systems-software.html>. Acesso em: 16 ago. 2016.

Gisgeography. 13 Free GIS Software Options: Map the World in Open Source. Disponível em: $<$ http://gisgeography.com/free-gis-software/>. Acesso em: 16 ago. 2016.

Leman, N.; Ramli, M. F.; Khirotdin, R. P. K. GIS-based integrated evaluation of environmentally sensitive areas (ESAs) for land use planning in Langkawi, Malaysia. Ecological Indicators, v. 61, p. 293-308, 2016.
Madeiros, J. S.; Câmara, G. Geoprocessamento para projetos ambientais. São José dos Campos: Instituto Nacional de Pesquisas Espaciais, Ministério da Ciência e Tecnologia, 2001.

Mahboubi, P.; Parkes, M.; Stephen, C.; Chan, H. M. Using expert informed GIS to locate important marine social-ecological hotspots. Journal of Environmental Management, v. 160, p. 342-352, 2015.

MMA - Ministério do Meio Ambiente. Geoprocessamento. 2016. Disponível em: $<$ http://www.mma.gov.br/governancaambiental/geoprocessamento>. Acesso em: 12 ago. 2016.

Pactwa, K.; Blachowski, J.; Górniak-Zimroz, J. GIS as a support tool in regional management of rock mineral resources: experiences from SW Poland. Procedia Earth and Planetary Science, v. 15, p. 488-494, 2015.

PMI - Project Management Institute. O que é gerenciamento de projetos? Newtown Square, PA: PMI, 2008. Disponível em: $<$ https://brasil.pmi.org/brazil/AboutUS/WhatIsP rojectManagement.aspx $>$. Acesso em: 12 maio 2016.

Reis, T. Gestão de projeto: o que é e para que serve? Rio de Janeiro, 2015. Disponível em: $<$ http://www.projectbuilder.com.br/blogpb/entry/conhecimentos/o-que-e-gestao-deprojetos-e-para-que-serve>. Acesso em: 12 maio 2016.

Vacca, A.; Loddo, S.; Melis, M. T.; Funedda, A.; Puddu, R.; Verona, M.; Fanni, S.; Fantola, F.; Madrau, S.; Marrone, V. A.; Serra, G.; Tore, C.; Manca, D.; Pasci, S.; Puddu, M. R.; Schirru, P. A GIS based method for soil mapping in Sardinia, Italy: a geomatic approach. Journal of Environmental Management, v. 138, p. 87-96, 2014.

Vafaeipour, M.; Zolfani, S. H.; Varzandeh, M. H. M.; Derakhti， A.; Eshkalag, M. K. Assessment of regions priority for implementation of solar projects in Iran: new application of a hybrid multi-criteria decision making approach. Energy Conversion and Management, v. 86, p. 653-663, 2014.

YSA - Youth Service America. 55 exemplos de projetos ambientais. Washington, DC, USA.

2009. Disponível em: <https://arcadeideias.files. wordpress.com/2015/06/55_exemplos_de_proje tos_ambientais.pdf $>$. Acesso em: 10 ago. 2016.

Informação da Licença: Este é um artigo Open Access distribuído sob os termos da Licença Creative Commons Attribution, que permite uso irrestrito, distribuição e reprodução em qualquer meio, desde que a obra original seja devidamente citada. 